\title{
Constancy scaling and the brackets illusion*
}

\author{
GORDON STANLEY \\ Eniversity of Melbourne \\ Parkille. I'ictoria. Australia 3052 \\ and \\ ARTHUR C. GRAESSER \\ Lniversity of California. San Diego. La Jolla, Calif. 92037
}

This experiment studied the effects of direction of bracket, height of bracket, and attachment or interspace on the apparent length of a line with bracket attachments. Results showed that outward brackets produced longer lines than inward brackets, interspacing produced longer lines, and increasing height of the brackets produced convergence of inward and outward functions. These results were considered partial support for the application of Day's general constancy explanation to the brackets illusion.

Recently there has been much interest in constancy scaling accounts of geometrical illusions. Perhaps the best known and most controversial work is that of Gregory (1963, 1965), who proposed to account for these size illusions in terms of misapplied constancy scaling produced by perspective cues for depth in the presence of constant-size retinal images. Subsequently, a number of experimental reports have shown that this theory is limited in scope (Day, 1965; Fisher, 1968). Much of Gregory's evidence for his theory was based on the Mueller-Lyer illusion, but Massaro \& Anderson (1970) have shown that the theory implies incorrect predictions about the three-dimensional version of this illusion.

More recently Day (1972) has proposed a general account of visual spatial illusions that does not rely on the perspective cue to depth. Day drew attention to the fact that there are many sources of information for depth. and proposed that all that is required for an illusion to occur is that there be a variation in one of these cues for depth in the presence of a fixed retinal image size.

While Day's (1972) paper did not specifically refer to the brackets illusion depicted in Fig. 1, he noted that a wide range of terminal attachments produce an illusion similar in form to the traditional arrows of the Mueller-Lyer illusion. He further pointed out that the outward-directed attachments generally produce an illusion three to four times that of the inner-directed elements, suggesting that the outward and inner attachment effects are probably separate illusions. On his theory. the outward effect is due to the size of the attachments. the inward effect being determined by the

\footnotetext{
*This study was completed while the first author was a visitor at the Center for Human Information Processing at the University of California. San Diego. Support came from NIMH Grant $\mathrm{MH}-15828$ to the Center. The authors are grateful to John Clavadetscher, Chervl Graesser. John Harris, and Al Stevens for assistance in data collection.
}

inner space defined by the inner attachments. Both the size of the attachments in the outward version and the inner space in the inward version are assumed to be distance stimuli which normally act to preserve size constancy.

As little previous work has been reported with the brackets illusion. the parameters of inward and outward at tachment, height of attachment, direct attachment or interspace between line and attachment, and line length were varied in a factorial design. The following predictions were made on the basis of Day's account: (1) There should be a significant inward-outward effect. the outward brackest producing longer mean estimates than the inner brackets. (2) As the inner space defined by the brackets is assumed to be the basis for the judgment with inward attachments, the presence of an interspace between the bracket and line should produce a longer line for these attachments. However, as size of the attachments is assumed to be the critical cue for the outward brackets. there should be no effect of interspace for the outward figure. In analysis of variance terms, this prediction implies a significant Interspace by Direction of Bracket interaction. (3) As larger size is a cue to nearer distance, the constancy-scaling mechanisms should produce judgments of smaller lines with taller brackets for the outward brackets. However. as inner space between the ends is the cue for the inner-directed attachments, varying size should have no effect for them, as the length of the inner space is constant, only its height being varied. Again, this prediction assumes a significant interaction, Height by Direction of Bracket.

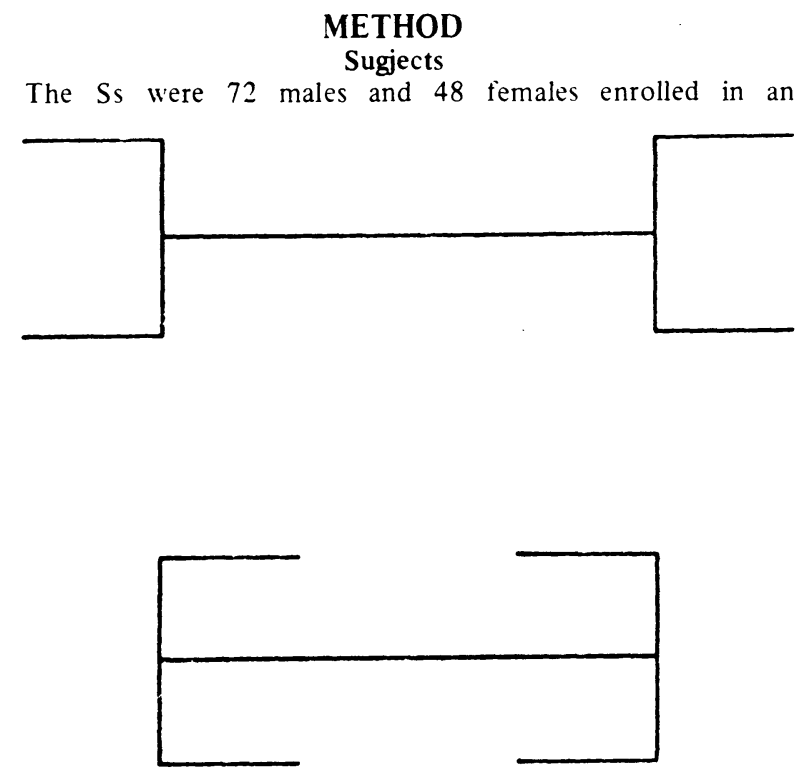

Fig. 1. The brackets illusion. The line with outward-directed brackets appears longer than the line with the inward-directed brackets. 


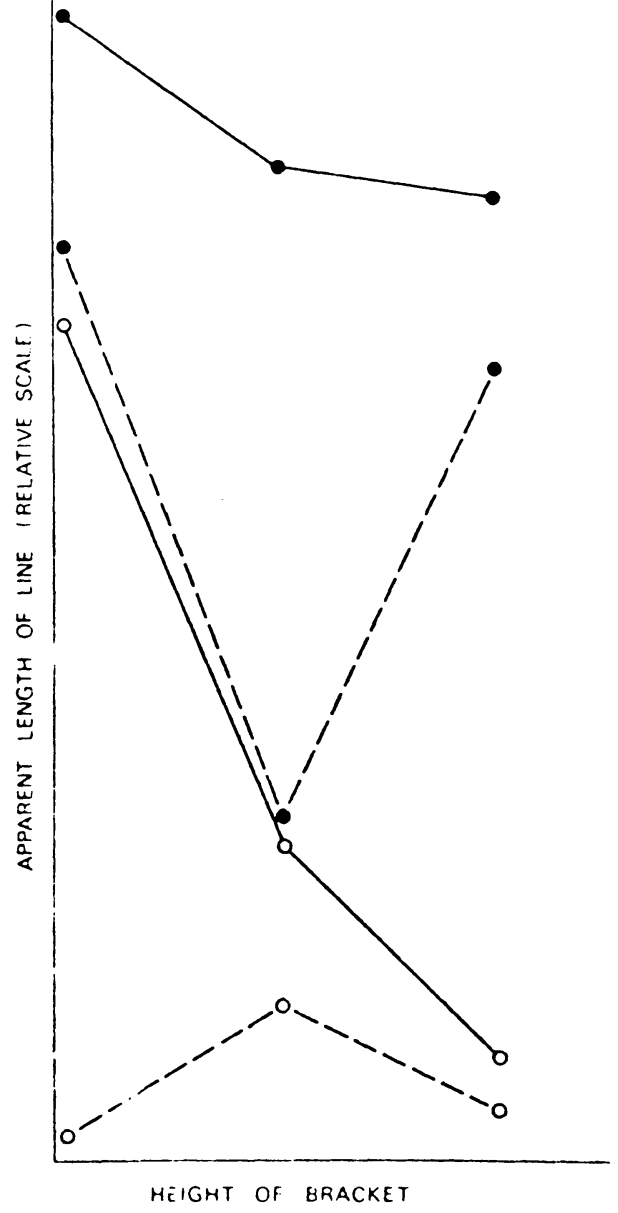

Fig. 2. Mean apparent length of center line plotted as a function of height of bracket. Solid lines represent outward-directed and broken lines represent inward-directed brackets. Open circles represent connected lines, closed circles represent interspaced lines.

introductory course at the University of California, San Diego. While most Ss had some knowledge of geometric illusions, they were not aware of the specific predictions of the experiment.

\section{Stimuli}

The stimuli were constructed with the appropriate values for the factorial design. Lines. 74. 92.110. and $128 \mathrm{~mm}$ long, were positioned centrally on a white sheet. $279 \times 216 \mathrm{~mm}$ in size. one line per sheet. The brackets had a width of $26 \mathrm{~mm}$ and varied in height. being 37.74 .111 . or $148 \mathrm{~mm}$ in height. For those cards on which there was an interspace between the line and the brackets. the interspace was $13 \mathrm{~mm}$. On half of the cards. the brackets faced inwards. on the other half. outwards.

\section{Procedure}

Small groups of Ss were presented with the stimuli one at a time, in random order. They were instructed to make magnitude estimates of the center horizontal straight line.

\section{Data Analysis}

Raw scores for each stimulus were transformed by dividing each person's estimate of that stimulus by the same person's estimate for the $128 \mathrm{~mm}$-long line with interspace and $148-\mathrm{mm}$-high outward brackets. As this transformation results in zero variance on estimates for the latter line. the level of 148-mm-high brackets was not used in subsequent analysis. The remaining data were subjected to analysis of variance according to a 2 by 2 by 3 by 4 within-S design. with direction of brackets. interspace bracket height. and line length as factors.

\section{RESULTS}

The mean length. averaged over all conditions, for the inward bracket lines was 0.767 . and that for outward bracket lines was 0.781 . the direction of brackets factor being significant $[F(1,119)=35.251, p<.01]$. The overall effect in interspacing appeared to be relatively constant for both inward and outward brackets; the mean difference between the mean inward brackets (averaged over all other conditions) for the connected and interspaced lines was 0.23 , and that for outward brackets was 0.25 . The Interspace by Direction of Bracket interaction was not significant $[F(1.119)=0.199, p>.05]$. However, there was a significant Height by Direction of Bracket interaction $[F(2,238)=3.851, \quad p<.05]$, and the third-order Interspace by Direction of Bracket by Height interaction was also significant $[F(2,238)=6.145, p<.01]$. Plots of the mean line lengths for each height of bracket, for direction of bracket, and for the connected-interspace factor are shown in Fig. 2. Contrary to prediction from Day's account, the interspace had an effect on outward bracket lines as well as on inward bracket lines. In his account, it was predicted that the effect of bracket height would be on the outward bracket lines, such lines being judged smaller with increasing height. As can be seen in Fig. 2, this prediction was confirmed. It should be noted that the effect was greater on the connected lines than on the lines with the interspace.

With the inward brackets, increasing height initially works in opposite directions for the connected and interspaced lines. For the connected line, it results in a small increase in length, whereas for the interspaced line, it produces a marked decrease in length. However, both the inward functions converge toward their respective outward functions with the largest height. It would appear that increasing height affects the judgment of line length over only a restricted range, and that for larger changes in size the discrepancy in length estimates between lines with inward and outward brackets decreases.

\section{DISCUSSION}

The present results partially support the application of Day's general constancy explanation to the brackets illusion. It could be argued that the convergence of the inward and outward functions for the largest height bracket helps define the limits over which such constancy scaling applies. The longer apparent lengths for the lines with interspaces shown in Fig. 2 would be accounted for on a constancy scaling theory by assuming that the interspace results in a judgment that such lines are further away and hence longer. This implication could be tested empirically by viewing luminous versions of the illusion in the dark.

\section{REFERENCES}

Day, R. H. Inappropriate constancy explanation of spatial 
distortions. Nature, 1965, 207. 891-893.

Day. R. H. Visual spatial illusions: A general explanation. Science. 1972, 175, 1335-1340.

Fisher. G. H. An experimental and theoretical appraisal of the inappropriate size-depth theories of illusions. British Journal inappropriate size-depth theories
of Psychology, $1968,59,373-383$.

Gregory, R. L. Distortion of visual space as inappropriate constancy scaling. Nature, 1963, 199, 678-680.
Gregory, R. L. Seeing in depth. Nature, 1965, 207. 16-19.

Massaro, D. W.. \& Anderson. N.H. A test of perspective theor of geometrical illusions. American Journal of Psychology. $1970,83,567-575$.

(Received for publication May 29.1973.)

\section{A comparison of presentation rates using a missing item probe test of immediate memory *:}

\section{NANCY S. ANDERSON $\dagger$ and V. DAVID BURNS \\ Lniversity of Maryland, College Park. Md. 20742}

Two experiments are reported in which presentation rates were compared using a "missing-item" probe technique for digit lists of 12 or 16 items. The results replicate previous studies (e.g., Waugh \& Norman, 1965) in that probability of recall was found to be a monotonically decreasing function of the number of intervening items and no significant differences were found between presentation rates. The results are interpreted as further evidence against a time decay hypothesis in immediate probed recall.

Several hypotheses have been proposed to explain the result that, when memory is tested at brief delays

* This research was supported in part by a grant from the Biomedical Sciences Support Center of the University of Maryland to the Center for Language and Cognition, and the computer time was supported through the facilities of the Computer Science Center.

tRequests for reprints should be sent to Nancy $S$. Anderson, Department of Psychology. University of Maryland. College Park. Md. 20742. following presentation, more recent list items are recalled with a higher probability than earlier items (recency effect). Brown (1958) argued that an item's trace strength decays over time. whereas other investigators (e.g., Waugh \& Norman, 1965) have concluded that interference resulting from the presentation of subsequent items is the primary cause of the effect. Of course, both factors could contribute to forgetting, as has been shown by Wicklegren (1970).

One method of distinguishing among the hypotheses is to compare performance across presentation rates. Unfortunately this comparison is complicated by the presence of differential time for rehearsal or encoding across presentation rates and by the possibility that order of recall interacts with presentation rate differences (Posner, 1964). The latter difficulty can be avoided by requiring the recall of only a single item within each list, e.g., by presenting a single-item probe and instructing Ss to recall the item which followed it in the original list (Waugh \& Norman. 1965). Waugh and Norman also instructed their Ss not to rehearse previous 